\title{
A Transmission Line Model for the Non-Invasive Evaluation of the Vascular Mechanical Properties at Level of the Brachial Artery
}

\author{
L T Mainardi(*),A Porta(*),D Lucini(\#),M Aquino(*),M Monaco(*),M Pagani(\#),S Cerutti(*) \\ $\left.{ }^{*}\right)$ Department of Biomedical Engineering, Polytechnic University, Milan, Italy \\ (\#) Medicina II, L.Sacco Hospital, University of Milan, Milan, Italy
}

\begin{abstract}
A method for the non-invasive measurement of the vascular properties at level of the brachial artery will be presented. The method uses non-invasive recordings of arterial blood pressure and flow carried out at different sites to estimate the transfer function between the two signals. A transmission-line model is used to fit the estimated transfer function and to extract the parameters of interest. Measurements of arterial compliance and peripheral resistance were extracted from normal subjects in different physiological experimental conditions.
\end{abstract}

\section{Introduction}

The physical state of the arterial tree may be investigated by measurements of the vascular input impedance [1][2][3]. In fact, changes in the input impedance have been documented in presence of vasoconstriction, altered mean arterial pressure, changes in the local compliance or arterial diseases [3].

Nevertheless, the need of invasive recordings and the lack of adequate simultaneous measurements of pulsatile blood pressure (BP) and blood flow (BF) signals from the same arterial site have limited the applicability of this approach for clinical purpose in humans [2].

In this paper, we investigate the property of the brachial artery in different physiological conditions by analyzing the characteristic of the Transfer Function (TF) between $\mathrm{BP}$ and $\mathrm{BF}$ waves which were non-invasively measured at different sites along the (right) arm. The pattern of the transfer function depends on the properties of the arterial tract between the two measurements as well as on the variation of the peripheral load. The estimated TFs are related to parameters of physiological interest (such as arterial compliance, peripheral resistance etc..) by means of a transmission line model [4][5]. The TF parameters were identificd by a weighed minimization of the difference between the estimated TF and the transmission line model one. Non-invasive measurements of arterial compliance, arterial and peripheral resistance were obtained through the transmission line parameters.

The proposed method can provide a non-invasive measurement of the physical state of the artery and indexes of clinical relevance for the study of the systemic arterial tree in humans.

\section{Methods}

\subsection{Estimation of the transfer function}

The transfer function, $I P(\omega)$, between $\mathrm{BP}$ and $\mathrm{BF}$ is estimated in frequency domain as follow [6]:

$$
H^{0}(\omega)=\frac{C_{p f}(\omega)}{S_{f}(\omega)}
$$

where $C_{p f}$ is the estimated cross-spectrum between the recorded blood flow and arterial pressure signals and $S_{f}$ the flow spectrum. From (1) the modulus of the TF becomes

$$
\left|H^{0}(\omega)\right|^{2}=\frac{S_{p}(\omega)}{S_{f}(\omega)} \cdot k^{2}(\omega)
$$

where $S_{p}$ is the estimated blood pressure spectrum and $k^{2}$ is the quadratic coherence between BP and BF. Equation (2) evidences that the estimated modulus is evaluated as the ratio of the two signal spectra, weighed by the coherence function. By taking into account the meaning of $k^{2}$, this estimate of $\left|H^{0}(\omega)\right|$ enlances the frequencies in which a tiglit linear relationship exists between the two signals, thus indicating where a consistent estimation of $\left|H^{0}(\omega)\right|$ can be obtained.

The value of $H^{\circ}(0)$ was estimated directly as the ratio of mean $\mathrm{BP}$ and mean $\mathrm{BF}$.

Both spectral and cross-spectral analysis were carried out by means of the Welch method. Each spectrum was estimated by considering 32 frames of 2048 samples, $50 \%$ oveilapped, and weighed by Hanning window [6]. 


\subsection{The model}

The brachial artery tract was modeled by a single, twoport, transmission line, closed on a resistive load $\left(R_{L}\right)$. The line tract was described by the following equations:

$$
\left[\begin{array}{l}
P_{1} \\
Q_{1}
\end{array}\right]=\left[\begin{array}{cc}
\cosh (\gamma d) & Z_{0} \sinh (\gamma d) \\
\frac{\sinh (\gamma d)}{Z_{0}} & \cosh (\gamma d)
\end{array}\right]\left[\begin{array}{c}
P_{2} \\
Q_{2}
\end{array}\right]
$$

where the $Q_{i}^{\prime}$ 's $(\mathrm{i}=1,2)$ and $P_{i}^{\prime}$ 's are the blood flows and the arterial pressures, respectively, at the two extremities of the line. $Z_{0}=\sqrt{\frac{R+j \omega L}{G+j \omega C}}$ is the characteristic impedance, $d$ is the vessel length and $\gamma=a+b \sqrt{\omega}+j \omega / v_{p}$ is the constant of propagation in which the velocity of propagation $v_{p}$ is evidenced. $R, L, C$ and $G$ are those reported in Fig 1 .

By assuming a purely resistive load $R_{L}$ at the end of the line (thus neglecting the compliance present in the small sized arterioles and capillaries), negligible pressure at the return line, and by taking into account (3), it is possible to express the transfer function $H(\omega)$ between $Q_{1}$ and $P_{2}$ (i.e. flow and pressure at different site) as

$$
H(\omega)=\frac{P_{2}}{Q_{1}}=Z_{0} \frac{\frac{R_{L}}{Z_{0}}}{\frac{R_{L}}{Z_{0}} \sinh (\gamma d)+\cosh (\gamma d)}
$$

Moreover, if we assume that $R<<\mathrm{j} \omega L$ and $G<<\mathrm{j} \omega C, Z_{0}$ becomes independent from $\omega$, being a simple function of $L$ and $C$

$$
Z_{0}=\sqrt{\frac{L}{C}}
$$

\subsection{Identification of the transmission line model}

Under the previous hypothesis, $H(\omega)$ is described by a set of parameters $\varphi=\left[\begin{array}{lllllll}a & b & v_{p} & R_{L} & Z_{0} & d\end{array}\right]$. The vector $\varphi^{0}$, identifying the transmission line which better fits the experimental curve $H^{0}$, is obtained by minimizing the following figure of merit:

$$
J=\frac{1}{N_{f}} \sum_{i=0}^{N_{f}}\left[\left(\frac{\left|H^{0}\left(\omega_{i}\right)\right|}{\sqrt{k^{2}\left(\omega_{i}\right)}}-\left|H\left(\omega_{i}\right)\right|\right) \cdot\left(k^{2}\left(\omega_{i}\right)\right)^{2}\right]^{2}
$$

$J$ includes the difference between the modulus of the FFT estimated TF, $\left|H^{0}(\omega)\right|$, and the model predicted ones, $|H(\omega)|$. This difference is weighed by quadratic coherence, which plays the same role as in (1). The summation is extended on the discrete frequency $\omega_{1}$ up to $N_{f}$ frequency points. $N_{f}$ was estimated as the upper limit at which $k^{2}$ becomes definitely lower than 0.5 .

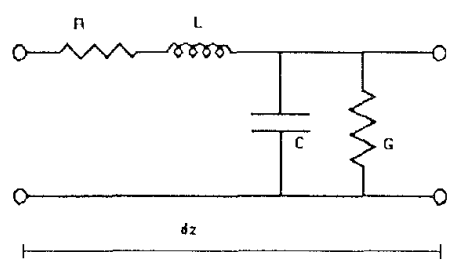

Figure 1. The elementary cell of the transmission line model.

The number of estimated parameters was reduced by imposing DC constrain. In fact, $R_{L}$ may be derived by the other parameter values and by the estimate of $H^{0}(0)$ :

$$
R_{L}=Z_{0} \frac{\frac{H^{0}(0)}{Z_{0}} \cdot \cosh (a d)}{1-\frac{H^{0}(0)}{Z_{0}} \cdot \sinh (a d)}
$$

The identification was carried out by means of an iterative, non-linear, least square procedure. Constrains were imposed to the coefficients in order to assure physiological meaning to the estimated parameters. The adopted limits are reported in table 1 .

$\begin{array}{cc}a & >0 \\ b & >0 \\ v_{p} & >0 \\ R_{L} & <H^{0}(0) \text { and }>0 \\ Z_{0} & \text { between } 4 \mathrm{~m} / \mathrm{s} \text { and } 8 \mathrm{~m} / \mathrm{s} \\ d & \text { between } 0.3 \mathrm{~m} \text { and } 0.6 \mathrm{~m}\end{array}$

Table 1. Constrains imposed to the parameters of the transmission line in the identification procedure (see text).

A quality index (QI) was finally evaluated in order to quantify the performance of the estimated model in the reproduction of the measured signals. The QI was defined as 


$$
\mathrm{QI}=\left(1-\frac{P_{e}}{P_{p}}\right) \times 100
$$

where $P_{\mathrm{p}}$ is the mean power of the measured BP and $P_{\mathrm{e}}$ is the power of the prediction error between the neasured and the model predicted BP. The predicted BP was obtained by feeding the estimated transmission line by the measured $B F$. The QI values range from 0 to 100 and represents the fraction of the BP power described by the model.

\section{Experimental protocol}

Ten normal subjects underwent the study. The experimental protocol was designed with different phases, including rest (R), mild exercise (EX) and recovery. In $\mathrm{R}$ the patient is lying on a bed in supine position. During exercise the patient was asked to cycle facing different loads $(10 \%, 20 \%$ and $30 \%$ of the nominal maximum load) remaining in supine position: a special cyclo-ergorneter, mounted on the bed, was used. The test ended with a 10 minutes of recovery.

During the tesst, ECG, arterial blood pressure and arterial blood flow were continuously recorded. Arterial blood pressure was non-invasively measured (via Omheda FINAPRES) at level of the mean finger, while arterial blood flow was recorded at level of omolateral Brachial artery via a Ultrasound Doppler flowmeter (Remco).

The Ultrasound Doppler flowmeter provides a measure of the mean blood flow velocity in the artery. To determine a flow measure expressed in $\mathrm{cm}^{3} / \mathrm{sec}$ it would be necessary to measure the average vascular diameters and other geometrical parameters. Since these are not available, the BF is calibrated in Ultrasound Doppler Shift units (UDsu), which are proportional to flow under the hypothesis that the unknown parameters are kept constant during the experiment.

The recorded signals were sampled at $300 \mathrm{~Hz}$ and stored on a $486 \mathrm{PC}$ for successive processing.

\section{Results}

Examples of FFT estimated transfer function $H^{0}(\omega)$ are shown in Fig. 2 (a)-(d) (continuous lines). Just a visual inspection evidences that the shape of the transfer function clanges passing from rest to excrcise. During rest, the modulus of the TF has a maximum at $\mathrm{DC}$, decreases until a few Hertz and an evident local maximum is present at around $7-8 \mathrm{~Hz}$. The phase, which slightly decreases up to $6-7 \mathrm{~Hz}$ has a sudden changes in presence of the local maximum in the modulus. During exercise, the modulus has more ripples because both pressure and flow spectrum are more concentrated due to the decrease of heart rate variability. In the same figure, the model estimated transfer function are superimposed (dotted lines). In both the experimental conditions the model seems to properly mimic the estimated transfer function.
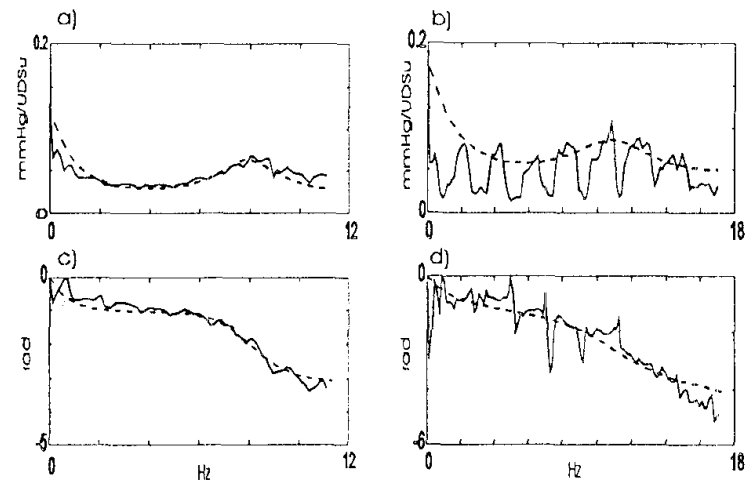

Figure 2 The estimated transfer functions (continuous line) during rest (a),(c) and exercise (b),(d). Both modula (upper panels) and phases (lower panels) are shown. The transmission line transfer functions are superimposed (dotted lines).

In particular, the model is able to capture both the presence of the local maximum in the modulus and the changes in the phase. The fit of the modulus is more precise as an effect of the definition of the figure of merit $J$ which minimizes the difference between the modula (see eq. 6). Nevertheless, an appropriate fitting of the phase is achieved.

For the same subject, the estimated vascular parameters are shown in Fig, 3 in correspondence to the different epochs of the test. Peripheral resistance is found to increase during exercise, as expected because the arm is not directly involved in the effort. Conversely arterial compliance decreases as a consequence of the increased stiffness of the artery during exercise [7]. The value of the characteristic impedance slightly increases in order to maintain an optimal match. Recovery restores the basal values of the parameters.

Finally, the performances of the model have been tested. The transmission line was fed by the measured blood flow and the predicted arterial pressure was calculated and compared with the measured ones. The two curves are shown in Fig. 5 in which the predicted blood pressure wave is plotted as a continuous line. The figure shows that the model is able to capture the mean shape of the BP, even if local small beat-to-beat disclosures in amplitude and shape can be observed. Such a disclosures may be due to the limits of the model (non-linear and visco-elastic 
effects) or to mechanisms of nervous system regulation [6]. Nevertheless the QI index was higher than $85 \%$, suggesting a good performance of the model.

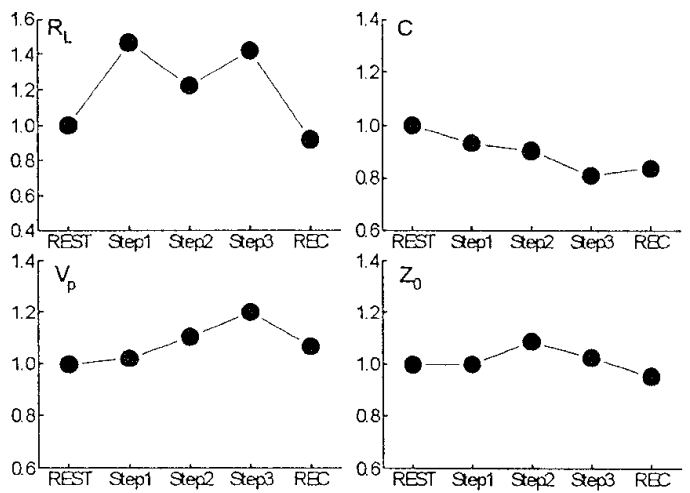

Figure 3. Example form one subject of estimated line transmission parameters during the different test phases. The plotted parameters: $\mathrm{R}_{\mathrm{L}}$ is the peripheral resistance, $\mathrm{C}$ is the artery compliance, $\mathrm{V}_{\mathrm{p}}$ is the wave propagation speed and $Z_{0}$ is the characteristic impedance. Step1,2 and $3=$ exercise at $10 \%, 20 \%$ and $30 \%$ of the maximum nominal load; $\mathrm{REC}=$ recovery. Parameters are normalized in respect to their basal values, in order to focus on the percentual variations.

\section{Discussion and Conclusions}

In this paper, we presented a non-invasive procedure to extract indexes of mechanical properties of the brachial artery. The procedure is based on the estimation of the transfer function between blood flow and arterial pressure which were measured at different sites along the brachial artery. The TF is successively fitted, in frequency domain, by a transmission line model in order to estimate the parameters of interest. The choice to fit the model in frequency domain allows to concentrate the fitting on the principal harmonic components of the signals, thus rcducing on the estimation the effects of additive wide-band noise or beat-to-beat local changes in the wave shapes.

Modeling the considered brachial artery tract by a single two-port transmission line seems an over simplification at a first sight. Nevertheless, results have shown that this simple model may adequately describe the system under study as evidenced by a good correspondences between real and estimated blood pressure waves.

The estimated transfer function do not have the direct physiological interpretations of the input impedance measurcment. However, when the transmission line model has been identified, the model may be used to estimate the value of vascular input impedance at beginning of the line, thus obtaining a corrected evaluation of vascular impedance from non-invasive $\mathrm{BF}$ and $\mathrm{AP}$ measurements from different site.

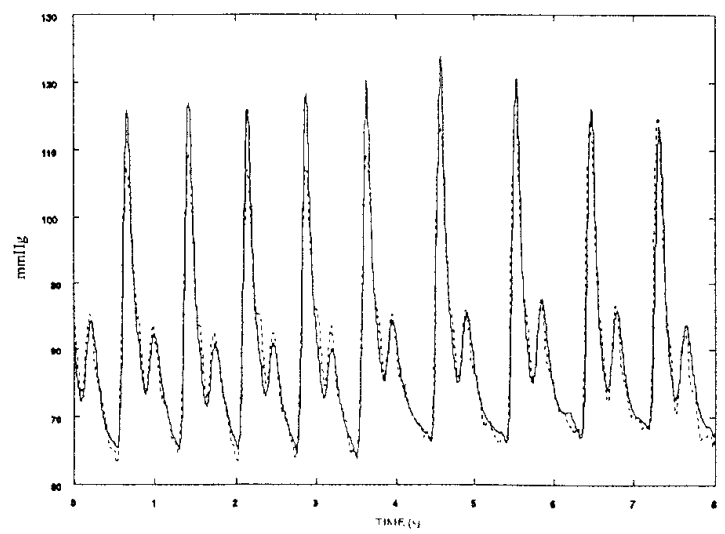

Figure 4. Real (dotted) and estimated (continuous) BP at level of the mean finger.

In conclusion, the presented approach seems to produce preliminary satisfactory results. The results suggest possible application in clinical routine even if the method needs further refinements and a deeper validation on a greater number of cases.

\section{References}

[1] J.P Murgo et al. Aortic Input Impedance in Man: Relationship to Pressure Waveforms. Circulation 1980, Vol. 62 , No. 1.

[2] W.W. Nichols, et al. Input Impedance of the Systemic Circulation in Man. Circulation Res., May 1977, Vol. 40, No. 5,

[3] M. F.O'Rourke. Vascular Impedance in Studies of Anterial and Cardiac Function, Physiol. Reviews, April 1982, Vol. 62, No 2 ,

[4] N. Westerhof, et al. Analog Studies of the Human Systemic Arterial Tree. I. Biomechanics 1969, Vol. 2, pp. 121-143.

[5] P. Segers, et al. Effect of Visco-Elastic Damping and Capillar Bed Model on the Peripheral Pressure Wave. In: Comp. In Card. 1995, Los Alamitos: IEEE Computer Society Press pp.681-684

[6] A.Porta, et al., Beat-to-beat variability of Microvascular Peripheral Resistance assessed with a Non-invasive Approach. In: Comp. In Card. 1995, Los Alamitos: IEEE Computer Society Press pp. 201-204

[7] J.P.Murgo at al. Effects of exercise on Aortic Input Impedance and Pressure wave Forms in Normal Humans. Circ. Res., March 1981, vol. 48, No.3. 